\title{
Development of sinus node disease in patients with AV block: implications for single lead VDD pacing
}

\author{
U K H Wiegand, F Bode, R Schneider, A Brandes, H Haase, H A Katus, J Potratz
}

\begin{abstract}
Objective-To investigate the incidence of sinus node disease after pacemaker implantation for exclusive atrioventricular (AV) block.

Design-441 patients were followed after VDD $(n=219)$ or DDD pacemaker (n = 222) implantation for AV block over a mean period of 37 months. Sinus node disease and atrial arrhythmias had been excluded by Holter monitoring and treadmill exercise preoperatively in 286 patients (group A). In 155 patients with complete AV block, a sinus rate above 70 beats/min was required for inclusion in the study (group B). Holter monitoring and treadmill exercise were performed two weeks, three months, and every six months after implantation. Sinus bradycardia below 40 beats/min, sinoatrial block, sinus arrest, or subnormal increase of heart rate during treadmill exercise were defined as sinus node dysfunction.
\end{abstract} Results-Cumulative incidence of sinus node disease was $0.65 \%$ per year without differences between groups. Clinical indi-

Accepted for publication 4 January 1999
519 patients with high degree AV block

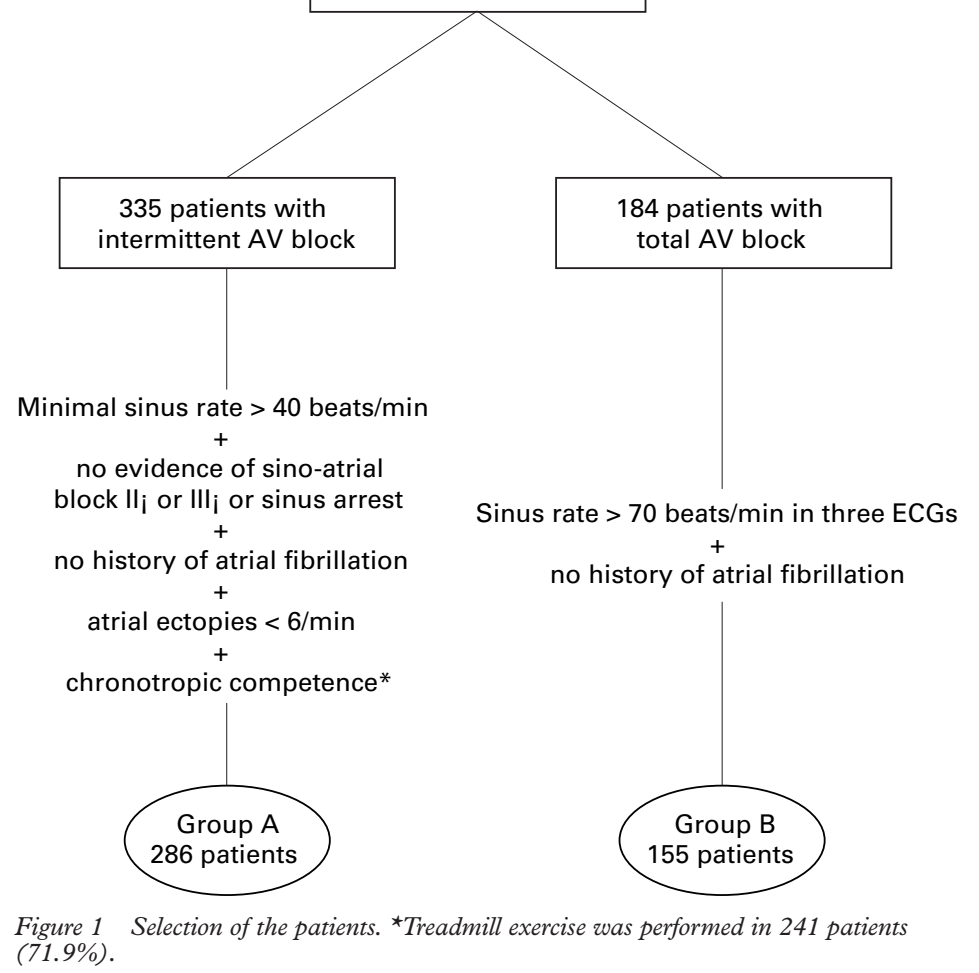

cators of sinus node dysfunction were sinus bradycardia below 40 beats/min in six patients $(1.4 \%)$, intermittent sinoatrial block in two $(0.5 \%)$, and chronotropic incompetence in five patients $(1.1 \%)$. Only one of these patients $(0.2 \%)$ was symptomatic. Cumulative incidence of atrial fibrillation was $2.0 \%$ per year, independent of the method used for the assessment of sinus node function and of the implanted device.

Conclusions-In patients undergoing pacemaker implantation for isolated AV block, sinus node syndrome rarely occurs during follow up. Thus single lead VDD pacing can safely be performed in these patients.

(Heart 1999;81:580-585)

Keywords: dual chamber pacing; sinus node disease; atrial fibrillation

Single lead VDD pacing ${ }^{1}$ is done in patients with high degree atrioventricular (AV) block and normal sinus node function, reducing time $^{2}$ and costs of implantation. The feasibility of atrial sensing through a floating dipole has been proven in several studies. ${ }^{3-5}$ However, chronotropic competence of the sinus node is crucial for maintenance of AV synchrony in patients receiving VDD pacemakers. Intermittent loss of AV synchrony may impair cardiac output $^{6}$ and cause a pacemaker syndrome, which is well known from VVI devices. ${ }^{7}$ Thus progressive sinus node disease may jeopardise the advantages of single lead VDD pacemaker implantation. The course of sinus node function, however, has not been systematically investigated in patients receiving a dual chamber pacemaker for isolated $\mathrm{AV}$ nodal disease.

The aim of our study was to evaluate the incidence of sinus node dysfunction and atrial arrhythmias in pacemaker recipients with $\mathrm{AV}$ block and normal sinus node function determined by Holter ECG.

\section{Methods}

PATIENTS

Between 1992 and 1997, 519 patients with high degree AV block were admitted to our hospital for pacemaker implantation. These patients were divided into two groups: patients with intermittent high degree AV block ( $\mathrm{n}=335$, group A) and patients with complete AV block who needed temporary pacing ( $n=184$, group B). In group A, patients were screened for sinus node disease by preoperative 
Table 1 Characteristics of patients. Group A: Holter electrocardiographic exclusion of sinus node disease; group $B$ : total $A V$ block fulfilling the sinus rate criterion of 70 beats/min

\begin{tabular}{|c|c|c|c|}
\hline & Group $A$ & Group B & All \\
\hline Number of patients & 286 & 155 & 441 \\
\hline Age (years) & $\begin{array}{l}68.0 \\
(13.4)\end{array}$ & $\begin{array}{l}71.3 \\
(12.2)\end{array}$ & $\begin{array}{l}69.1 \\
(13.1)\end{array}$ \\
\hline Sex (male \%) & 71.3 & 72.3 & 71.9 \\
\hline Pacemakers (VDD/DDD) & $142 / 144$ & $77 / 78$ & $219 / 222$ \\
\hline \multicolumn{4}{|l|}{ Indications } \\
\hline AV block III (\%) & 31.2 & 100.0 & 55.4 \\
\hline AV block II (\%) & 44.4 & & 28.8 \\
\hline Trifascicular block (\%) & 24.4 & & 15.8 \\
\hline \multicolumn{4}{|l|}{ Concomitant disease } \\
\hline $\begin{array}{l}\text { Coronary artery disease } \\
(\%)\end{array}$ & 40.6 & 43.2 & 41.5 \\
\hline \multicolumn{4}{|l|}{ Heart failure NYHA II \& } \\
\hline III (\%) & 28.3 & 33.5 & 30.2 \\
\hline Follow up (months) & $\begin{array}{l}37.2 \\
(19.1)\end{array}$ & $\begin{array}{l}35.8 \\
(19.8)\end{array}$ & $\begin{array}{l}36.7 \\
(19.4)\end{array}$ \\
\hline
\end{tabular}

There was no significant difference between the groups. Age and follow up periods are given as mean (SD).

NYHA, New York Heart Association functional class.

Table 2 Percentage of patients taking antiarrhythmic drugs

\begin{tabular}{lcc}
\hline Drug & Implantation & Follow up \\
\hline Amiodarone & 1.1 & 5.4 \\
B Blockers & 8.6 & 38.3 \\
Calcium antagonists & 0.0 & 2.3 \\
Digitalis & 3.9 & 16.3 \\
Flecainide & 0.0 & 0.5 \\
Sotalol & 1.8 & 7.0 \\
No antiarrhythmic drugs & 85.9 & 48.8
\end{tabular}

Holter monitoring and treadmill exercise. A sinus bradycardia below 40 beats/min, second or third degree sinoatrial block, intermittent sinus arrest, frequent atrial ectopic beats (more than five a minute), and chronotropic incompetence led to exclusion from the study (fig 1). In group $\mathrm{B}$, a sinus rate above 70 beats/min documented in three ECGs during at least 12 hours after admission was necessary for inclusion in the study. A history of atrial fibrillation or flutter led to exclusion from the study. Patients either received single lead VDD pacemakers $(n=219)$ or DDD devices with fixed bipolar atrial leads



Figure 2 Cumulative incidence of sinus node dysfunction. There was no significant difference between patients included by Holter electrocardiographic exclusion of sinus node disease (group A) and patients with total AV block fulfilling the sinus rate criterion of 70 beats/min (group B). $(n=222)$. Patient characteristics are described in table 1 . In all, $51.2 \%$ of patients received antiarrhythmic drugs at the time of implantation or during further follow up (table 2).

\section{DEVICES AND LEADS}

Four different VDD devices with their complementary tri- or quadripolar leads were implanted: Dromos 03 or SL with SL 60 UP (Biotronik, Berlin, Germany n = 24), Maestro with Polysafe (CCS Inc, Palmcoast, California, USA $n=11$ ), Saphir 600 with Brilliant (Vitatron, Diereu, The Netherlands $n=132)$, and Thera VDD with CapSure 5032 (Medtronic, Minneapolis, Minnesota, USA $n=52$ ). In the DDD subgroup, the following devices were included: Paragon II/III (Pacesetter AB, Solna, Sweden $\mathrm{n}=117$ ), Physios 1 (Biotronik, $\mathrm{n}=10$ ), Ruby 700 (Vitatron, $\mathrm{n}=71$ ), and Thera D (Medtronic, $\mathrm{n}=24$ ), all with bipolar atrial screw in leads. Maximal programmable atrial sensitivity ranged from 0.07 to $0.18 \mathrm{mV}$ in VDD pacemakers and from 0.18 to $0.50 \mathrm{mV}$ in DDD devices.

\section{ECHOCARDIOGRAPHY}

Cross sectional echocardiography was performed routinely in all patients before pacemaker implantation, including measurement of atrial $(n=410)$ and end diastolic left ventricular diameter in the parasternal view $(\mathrm{n}=387)$ as well as ejection fraction by the method of Simpson $(\mathrm{n}=343)$.

FOLLOW UP

All pacemakers were programmed to maximum atrial sensitivity immediately after implantation. Pacemaker interrogation, 12 lead ECG records for one minute, 24 hour Holter monitoring, and treadmill exercise were performed two weeks and three months after implantation, and then at six month intervals. Immediately after implantation, the intervention rate of the pacemaker was set to 40 beats/ min, facilitating the detection of sinus bradycardia. A short AV delay of $125 \mathrm{~ms}$ was programmed to guarantee ventricular pacing. Additional features such as mode switch or rate smoothing algorithms and AV or rate hysteresis were inactivated during Holter monitoring and treadmill exercise. Mean (SD) follow up time was 36.7 (19.4) months.

HOLTER MONITORING

We used a two channel recorder with stimulus detection (Tracker 3, Reynolds Medical, Hertford, UK); mean recording time was 22.4 hours (17.3 to 25.2 ). The tapes were analysed beat to beat at low speed by an experienced investigator using a Pathfinder 4 unit (Reynolds Medical). Reliable P wave detection was assured by visualisation of the ECG before starting the Holter records. Holter records were analysed for mean, minimum, and maximum heart rate, signs of sinus node dysfunction, and atrial arrhythmias.

TREADMILL EXERCISE

Treadmill exercise was performed using the Bruce protocol; indications for termination 


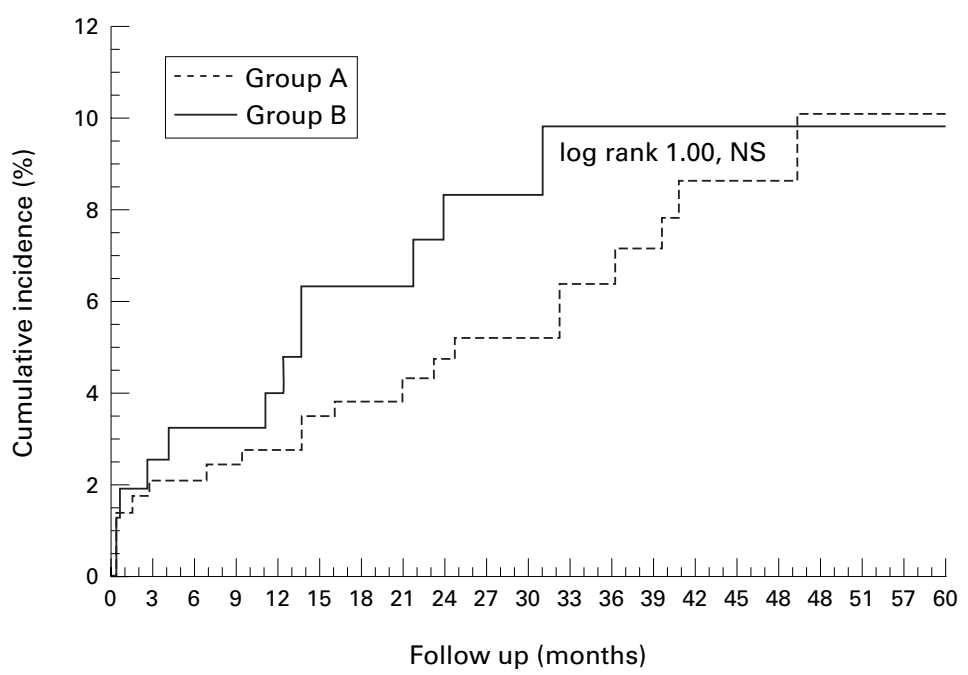

Figure 3 Cumulative incidence of atrial fibrillation. There was no significant difference between patients included by Holter electrocardiographic exclusion of sinus node disease (group A) and patients with total AV block fulfilling the sinus rate criterion of $70 \mathrm{beats} / \mathrm{min}$ (group B).
Atrial oversensing was assumed if rapid ventricular pacing was associated with artefacts visible on the ECG tape.

ANALYSIS OF DATA

Cumulative incidences of sinus node disease and atrial fibrillation were displayed by Kaplan-Meier curves. Comparison between group A and B, as well as between VDD and DDD devices, was performed by a log-rank test. Change of heart rate during follow up was analysed by a one way analysis of variance (ANOvA) with post hoc Scheffé analysis. Incidence of sinus node dysfunction in patients with and without antiarrhythmic treatment was compared by the $\chi^{2}$ test. Heart rates and incidence of supraventricular ectopic beats at time of implantation as well as echocardiographic variables were correlated with further development of sinus node disease or atrial fibrillation. Multivariate analysis was performed to isolate independent preoperative predictors for sinus node disease and atrial fibrillation.

were progressive chest pain, dyspnoea, exhaustion, dizziness, complex ventricular arrhythmias, submaximal work load $(0.8 \times(220-$ age)), or patient's request. Owing to the high incidence of structural heart disease, submaximal work load was not expected to be reached in the majority of patients. Therefore metabolic equivalents were estimated with help of a nomogram at each minute of exercise. Chronotropic incompetence was assumed if the heart rate was more than 2 SD below normal rate. ${ }^{8}$

SINUS NODE DISEASE

Sinus node dysfunction was defined by sinus bradycardia below 40 beats/min, second or third degree sinoatrial block, and intermittent sinus arrest. A sinus node syndrome was assumed if at least one of these events coincided with symptoms such as fatigue or dyspnoea.

SENSING DEFECTS

Atrial undersensing was defined by a $\mathrm{P}$ wave not followed by ventricular stimulation after the programmed AV delay (125 ms) or by atrial pacing more than $50 \mathrm{~ms}$ after onset of a $\mathrm{P}$ wave.

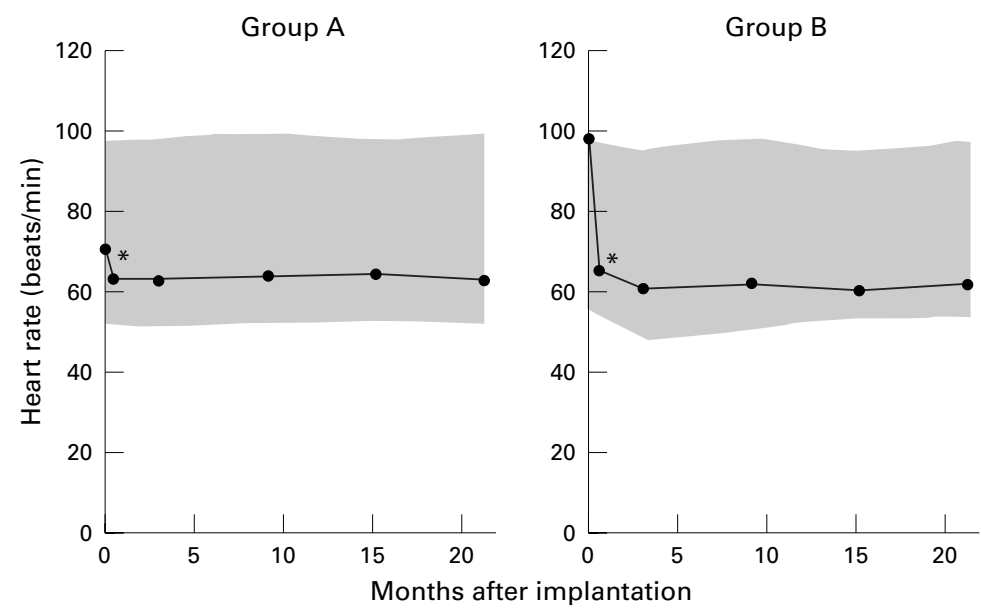

Figure 4 Mean, maximum, and minimum heart rate during Holter monitoring. $A$ significant decrease of mean heart rate was observed after implantation of pacemaker.

\section{Results}

ATRIAL SENSING

Intermittent atrial undersensing was observed in $22.8 \%$ of patients with VDD pacemakers and $20.3 \%$ with DDD devices (NS). There was also no difference between patients with (group A, 21.3\%) and without (group B, 21.9\%) preoperative Holter ECG exclusion of sinus node disease. Two patients with VDD pacemakers $(0.9 \%)$ who developed sustained and symptomatic atrial undersensing received an additional atrial screw in lead. Sustained atrial undersensing was caused by dislodgement of the atrial lead in three patients with DDD pacemakers $(1.4 \%)$.

INCIDENCE OF SINUS NODE DISEASE AND ATRIAL FIBRILLATION

After five years of follow up, the cumulative incidence of sinus node disease was $2.6 \%$ in patients with preoperative exclusion of sinus node disease by Holter ECG and exercise testing (group A) and $4.6 \%$ in patients fulfilling the criterion of a preoperative sinus rate above 70 beats/min (group B) (fig 2). These incidences were not significantly different. Intermittent sinoatrial block occurred in two patients $(0.5 \%)$, sinus bradycardia below 40 beats/min in six patients $(1.4 \%)$, and chronotropic incompetence was observed in five patients $(1.1 \%)$. Three patients showed more than one sign of sinus node dysfunction, but only one of these patients suffered from symptoms $(0.2 \%)$. The incidence of sinus node disease was comparable in VDD and DDD pacemakers $(1.8 \%$ v $2.3 \%$, NS). Sinus node dysfunction was not documented in any patient within the first two weeks of follow up.

The cumulative incidence of atrial fibrillation and flutter was $2.0 \%$ per year both in patients with preoperative Holter electrocardiographic exclusion of sinus node disease (group A) and in those without (group B, fig 3). There was also no significant difference in frequency of atrial tachyarrhythmias between 
Table 3 Heart rates, frequency of atrial ectopic beats, and echocardiographic variables for patients with and without later occurrence of sinus node disease (SND)

\begin{tabular}{|c|c|c|c|c|}
\hline & \multicolumn{2}{|l|}{ With SND } & \multicolumn{2}{|l|}{ Without SND } \\
\hline & Mean $(S D)$ & $95 \% C I$ & Mean (SD) & $95 \% C I$ \\
\hline \multicolumn{5}{|l|}{ Holter ECG $(n=286)$} \\
\hline Mean heart rate (beats/min) & $69.5(13.1)$ & 53.2 to 85.8 & $70.7(12.6)$ & 69.2 to 72.2 \\
\hline Minimum heart rate (beats/min) & $55.4(16.8)$ & 34.5 to 76.3 & $56.1(11.3)$ & 54.8 to 57.4 \\
\hline Maximum heart rate (beats/min) & $105.6(27.5)$ & 71.4 to 139.8 & $104.8(22.9)$ & 102.1 to 107.5 \\
\hline Range of heart rate (beats/min) & $40.2(19.4)$ & 16.1 to 64.3 & $38.7(14.7)$ & 37.0 to 40.4 \\
\hline Atrial ectopic beats (n) & $331(178)$ & 188 to 475 & $355(143)$ & 338 to 372 \\
\hline \multicolumn{5}{|l|}{ Exercise testing $(\mathrm{n}=208)$} \\
\hline Peak heart rate (beats/min) & $110.6(27.5)$ & 72.5 to 148.7 & $112.7(31.3)$ & 108.4 to 117.0 \\
\hline \multicolumn{5}{|l|}{ Echocardiography } \\
\hline Left atrial diameter $(\mathrm{mm})(\mathrm{n}=410)$ & $38.9(7.9)$ & 32.4 to 45.4 & $36.9(6.2)$ & 36.3 to 37.5 \\
\hline $\operatorname{LVEDD}(\mathrm{mm})(\mathrm{n}=387)$ & $54.7(6.9)$ & 48.5 to 60.9 & $53.1(5.8)$ & 52.5 to 53.7 \\
\hline Ejection fraction $(\%)(n=343)$ & $56.1(11.4)$ & 45.9 to 66.3 & $57.4(9.2)$ & 56.4 to 58.4 \\
\hline \multicolumn{5}{|l|}{ Concomitant disease $(n=441)$} \\
\hline Coronary artery disease & $33.3 \%$ & & $38.9 \%$ & \\
\hline Heart failure & $22.2 \%$ & & $28.9 \%$ & \\
\hline
\end{tabular}

Table 4 Heart rates, frequency of atrial ectopic beats, and echocardiographic variables for patients with and without later occurrence of atrial fibrillation $(A F)$

\begin{tabular}{|c|c|c|c|c|c|}
\hline & \multicolumn{2}{|l|}{ With $A F$} & \multicolumn{2}{|l|}{ Without AF } & \multirow[b]{2}{*}{$p$ Value } \\
\hline & Mean (SD) & $95 \% C I$ & Mean (SD) & $95 \% C I$ & \\
\hline \multicolumn{6}{|l|}{ Holter ECG $(n=286)$} \\
\hline Mean heart rate (beats/min) & $71.1(14.4)$ & 64.4 to 77.8 & $70.6(13.2)$ & 69.0 to 72.2 & NS \\
\hline Minimum heart rate (beats/min) & $54.1(17.3)$ & 46.0 to 62.2 & $56.2(16.1)$ & 54.3 to 58.1 & NS \\
\hline Maximum heart rate (beats/min) & $103.9(29.3)$ & 90.2 to 117.6 & $104.9(27.5)$ & 101.6 to 108.2 & NS \\
\hline Range of heart rate (beats $/ \mathrm{min}$ ) & $49.8(18.2)$ & 41.3 to 58.3 & $48.7(21.7)$ & 46.1 to 51.3 & NS \\
\hline Atrial ectopic beats (n) & $295(155)$ & 223 to 367 & $362(129)$ & 346 to 378 & NS \\
\hline \multicolumn{6}{|l|}{ Exercise testing $(\mathrm{n}=208)$} \\
\hline Peak heart rate (beats/min) & $108.4(32.7)$ & 90.4 to 126.4 & $113.0(26.4)$ & 109.3 to 116.7 & NS \\
\hline \multicolumn{6}{|l|}{ Echocardiography } \\
\hline Left atrial diameter $(\mathrm{mm})(\mathrm{n}=410)$ & $43.1(9.7)$ & 39.3 to 46.9 & $36.4(5.4)$ & 35.9 to 36.9 & $<0.001$ \\
\hline $\operatorname{LVEDD}(\mathrm{mm})(\mathrm{n}=387)$ & $54.5(10.9)$ & 49.9 to 59.1 & $53.0(6.2)$ & 52.4 to 53.6 & NS \\
\hline Ejection fraction $(\%)(n=343)$ & $52.7(14.4)$ & 46.3 to 59.1 & $57.7(8.8)$ & 56.7 to 58.7 & $<0.001$ \\
\hline \multicolumn{6}{|l|}{ Concomitant disease $(n=441)$} \\
\hline Coronary artery disease & $41.2 \%$ & & $39.7 \%$ & & NS \\
\hline Heart failure & $52.9 \%$ & & $38.8 \%$ & & $<0.01$ \\
\hline
\end{tabular}

CI, confidence interval; LVEDD, left ventricular end diastolic diameter.

VDD and DDD pacemakers $(6.8 \%$ \% $7.7 \%$, NS). Intermittent atrial fibrillation occurred in 21 patients $(4.8 \%)$ and was controlled with antiarrhythmic drugs in $17(3.8 \%)$ without development of symptomatic sinus bradycardia. In addition, mode switch algorithms were activated in 15 patients (3.4\%). In five patients $(1.1 \%)$, persistent atrial fibrillation could be converted to sinus rhythm by dc shock. Six pacemakers were programmed to $\mathrm{VVI}(\mathrm{R})$ mode owing to refractory atrial fibrillation $(1.4 \%)$.

Atrial fibrillation was associated with sinus bradycardia below 40 beats $/ \mathrm{min}$ in two patients with VDD pacemakers $(0.4 \%)$. In two patients each with VDD and DDD pacemakers, it coincided with intermittent atrial undersensing $(0.9 \%)$. Induction of atrial fibrillation owing to asynchronised atrial pacing after an undersensed $\mathrm{P}$ wave was observed in one patient with a DDD device.

HEART RATE DURING FOLLOW UP

Average heart rate decreased significantly within in the first two weeks after implantation (fig 4). This was predominantly caused by $\beta$ blocker treatment for coronary artery disease initiated after pacemaker implantation. In this subgroup, mean and minimum heart rate markedly decreased compared with the pretreatment values: mean (SD) 71.5 (11.6) $v$ 57.4 (10.5) beats/min, and 56.7 (11.1) v 49.8 (10.5) beats/min $(p<0.001)$, respectively. Sinus node disease did not occur more often in patients treated with antiarrhythmic drugs or $\beta$ blockers as compared with patients who did not receive negative chronotropic drug treatment $(2.2 \%$ v $1.9 \%$, NS).

PREDICTION OF SINUS NODE DISEASE AND ATRIAL FIBRILLATION

Occurrence of sinus node disease could not be predicted by preoperative Holter monitoring and exercise testing, or by the need for antiarrhythmic treatment (table 3). Patients who developed atrial fibrillation during follow up showed a larger left atrial diameter and a reduced left ventricular ejection fraction (table 4).

\section{Discussion}

The implantation of single lead VDD pacemakers has proved to be an excellent alternative to conventional dual chamber pacing, 
achieving a high degree of AV synchrony ${ }^{4-9}$ and low complication rates. ${ }^{10}$ Chronotropic incompetence, however, results in a markedly reduced efficacy of VDD pacing. Therefore, it is necessary to estimate the risk of developing sinus node dysfunction in these pacemaker recipients. Though AV block is well known to occur in patients with sick sinus syndrome, ${ }^{11} 12$ the occurrence of sinus node disease has not been systematically evaluated in patients with AV block. Our study proves that there is a low incidence of sinus node disease during follow up of patients with AV block and normal preoperative sinus node function.

PREOPERATIVE ASSESSMENT OF SINUS NODE FUNCTION

Holter electrocardiography is the most useful tool for evaluation of sinus node function. ${ }^{13}{ }^{14}$ The most relevant manifestations of sinus node disease, such as sinus bradycardia, sinoatrial block, sinus arrest, and atrial arrhythmias, can be documented by this method. Exercise testing is the method of choice for additional evaluation of chronotropic competence of sinus node. ${ }^{15}$ We used both Holter electrocardiography and exercise testing for preoperative exclusion of sinus node disease. However, in pacemaker dependent patients with complete AV block neither method is feasible. Therefore alternative methods have to be used to evaluate sinus node function. Atropine testing is of limited value as it achieves only a low sensitivity for determination of chronotropic competence. ${ }^{16}$ The diagnostic efficacy of this test may even be worse in intensive care patients who often have a high sympathetic tone. Determination of sinus node recovery time has also been shown to have low sensitivity for the diagnosis of sinus node syndrome. ${ }^{17}{ }^{18}$ Transient single lead VDD pacing may facilitate the evaluation of sinus node function in patients with complete AV block. However, costs and potential risks do not justify this approach for diagnostic purposes. In our study, simple criteria such as a history without atrial tachyarrhythmias and a sinus rate greater than 70 beats/min sufficiently described normal sinus node function in patients with complete AV block. All patients showed normal heart rates in the initial Holter ECG after implantation. In addition, patient selection by these criteria did not increase the risk of sinus node syndrome during further follow up. Thus where transient pacing for symptomatic high degree AV block is required, the patient's history and a simple sinus rate criterion at rest are useful in making a decision about whether a single lead VDD pacemaker can be implanted.

DEVELOPMENT OF SINUS NODE DYSFUNCTION

Sinus node function is impaired in $0.2 \%$ of an otherwise healthy population ${ }^{19}$ and in more than $10 \%$ of patients undergoing pacemaker implantation for AV block..$^{21}$ In our study, $21 \%$ of the initially screened patients were excluded preoperatively because of signs of sinus node dysfunction. In patients with isolated $\mathrm{AV}$ nodal disease at the time of pacemaker implantation, the incidence of sinus node dysfunction was low, at $0.7 \%$ per year. During a mean follow up of 37 months, only one of our 441 patients developed a symptomatic sinus node syndrome requiring DDDR pacemaker implantation. This finding is in agreement with results from patients with isolated AV block, in whom a deterioration of sinus node function did not occur during follow up. ${ }^{22}$ Even though antiarrhythmic or $\beta$ blocker treatment was started in half of our patients during follow up, the overall incidence of chronotropic incompetence was only $1.1 \%$. Completely divergent from these findings, Gwinn et al observed a $56 \%$ incidence of chronotropic incompetence in a small study of 27 patients paced for AV block. ${ }^{23}$ Chronotropic incompetence was increased in this study if pacemaker therapy lasted longer than four years. Apart from the small number of patients and divergent methods of preoperative evaluation in that study, the definitions of chronotropic incompetence also differed between the studies. Owing to a high incidence of concomitant cardiopulmonary disease, the maximum rate criterion ${ }^{24}$ used in Gwinn's study is mostly unattainable in a pacemaker population. Moreover, this method is not acceptable for patients with coronary artery disease. ${ }^{25}$ Therefore we defined chronotropic competence by a nomogram applicable to any work stage which was introduced by Wiens et al. ${ }^{8}$

Thus sinus node dysfunction is unlikely to occur in patients with AV block and regular sinus node function at the time of pacemaker implantation. In these patients, the data do not suggest the need for the prophylactic implantation of a rate adaptive DDD pacemaker, even if it is intended to treat the patient with negative chronotropic drugs, in particular $\beta$ blockers.

\section{ATRIAL FIBRILLATION}

Compared with ventricular demand pacing, atrial pacing reduces the incidence of atrial fibrillation and consecutive thromboembolic events in patients with sinus node disease. ${ }^{25}$ This beneficial effect of atrial pacing on atrial stability is mainly explained by overdrive suppression of atrial ectopic beats which occurs more often during sinus bradycardia. In patients with AV block and normal sinus node function, we found a considerably lower incidence of atrial arrhythmias. It is unclear at present whether the incidence of atrial arrhythmias is influenced by the implantation of an atrial lead. Of the $7.1 \%$ of patients with intermittent atrial fibrillation, only a small minority $(0.4 \%)$ showed an association of atrial fibrillation with sinus node disease. It is only in this group that bifocal stimulation might have been beneficial. The medical treatment of atrial arrhythmias could be facilitated by the presence of an atrial back up stimulation. On the other hand, atrial fibrillation can be induced by $P$ wave undersensing followed by asynchronised atrial pacing. ${ }^{26}$ Though follow up studies of patients with isolated AV block suggest lower rates of atrial fibrillation in VDD pacemakers than in DDD devices- $0.4 \%$ to $2.2 \%$ per year $^{34} v 1.0 \%$ to $3.8 \%$ per year ${ }^{27} 28$-we found a similar incidence in both groups by direct 
comparison. In the light of these data, the availability of algorithms that avoid a fast ventricular conduction of these atrial arrhythmias is much more important than the choice either of a VDD or a DDD device. In addition, the ability of use rate adaptive VVIR pacing should be available in pacemakers implanted for complete AV block. Algorithms for automatic switching from the VDD to a VVIR mode have been shown to work even in the low atrial amplitudes perceived through a floating dipole. ${ }^{29}$ Particularly, patients with depressed left ventricular function or increased left atrial diameter, in whom the development of atrial fibrillation is more likely, ${ }^{30}$ should be considered for this feature.

\section{LIMITATIONS}

A crucial point of every study dealing with sinus node function is that the definition of chronotropic incompetence varies considerably. The maximum heart rate criterion ${ }^{15}$ is not useful in patients with structural heart disease. Spiroergometric determinations of chronotropic competence ${ }^{31}$ are more exact but often not well tolerated in older patients. By the definitions used in our study, chronotropic competence could be evaluated by low level exercise which is tailored to the majority of pacemaker recipients.

The recognition of sinus bradycardia in pacemaker patients is difficult. An intervention rate of 40 beats/min was the lowest programmable rate in several devices, and appeared to be the lowest acceptable rate in pacemaker dependent patients to avoid discomfort when atrial sensing defects or severe bradycardia occurred. Therefore the rate criterion for detection of sinus node dysfunction was restricted to 40 beats $/ \mathrm{min}$.

\section{CONCLUSIONS}

The incidence of sinus node disease is low in patients undergoing pacemaker implantation for isolated AV nodal disease. In these patients, atrial fibrillation is not usually associated with sinus node dysfunction, and DDD pacing has not been shown to reduce its incidence. A Holter ECG should be performed routinely before implantation of a VDD pacemaker. However, in case of complete AV block, a sinus rate $>70$ beats/min is a sufficient criterion for exclusion of sinus node disease. Thus single lead VDD pacemaker treatment is an excellent option for patients with AV block and a normal preoperative sinus node function.

1 Antonioli GE, Grassi G, Baggioni GF. A single P-sensing ventricle stimulating lead driving a VAT generator. In: Mere $\mathrm{C}$, ed. Proceedings of the sixth world symposium on cardiac pacing, Montreal, 1979:34-9.

2 Wiegand UKH, Taubert G, Schneider R, et al. Single-lead VDD-pacemakers in patients with AV-block. An alternative to DDD-pacemaker-systems. Seventh European symposium on cardiac pacing and electrophysiology. Bologna: Monduzzi Edditore, 1995:37-41.
3 Curcio J, the Multicenter Study Group. A multicenter evaluation of a single-pass lead VDD system. PACE 1991; 14:434-42.

4 Crick JC. European Multicenter prospective follow-up study of 1002 implants of a single lead VDD pacing system. The Multicenter Study Group. PACE 1991;14: 1742-4.

5 Folino AF, Buja G, Ruzza L, et al. Long-term follow-up of patients with single lead VDD stimulation. PACE 1994;17: $1854-8$

6 Rediker DE, Eagle KA, Homma S, et al. Clinical and hemodynamic comparison of VVI versus DDD pacing in patients with DDD pacemakers. Am f Cardiol 1988;61: 323-9.

7 Schuller H, Brandt J. The pacemaker syndrome: old and new causes. Clin Cardiol 1991;14:336-40.

8 Wiens RD, Lafia P, Marder CM, et al. Chronotropic incompetence in clinical exercise testing. Am f Cardiol 1984;54: $74-8$.

9 Res JC, Reijsoo FJ, van Woersem RJ, et al. P wave recognition and atrial stimulation with fractally iridium coated VDD single pass leads. PACE 1994;17:1883-8.

10 Wiegand UKH, Scneider R, Bode F, et al. Does the implantation of single lead VDD-devices reduce the complication rate in dual chamber pacemakers? [abstract] $P A C E$ 1997;20:1563.

11 Elshot SR, el Gama MI, Tielen KH, et al. Incidence of atrioventricular block and chronic atrial flutter/fibrillation after implantation of atrial pacemakers; follow-up of more than ten years. Int f Cardiol 1993;38:303-8.

12 Swiatecka G, Sielski S, Wilczek R, et al. Atrioventricular conduction disturbances in patients with sinoatrial node disease and atrial pacing. PACE 1992;15:2074-6.

13 Ferrer MI. The sick sinus syndrome. Circulation 1973:47: $635-41$

14 Sneddon JF, Camm AJ. Sinus node disease. Current concepts in diagnosis and therapy. Drugs 1992;44:728-37.

15 Hammond HK, Froelicher VF. Normal and abnormal heart rate responses to exercise. Prog Cardiovasc Dis 1985;27: $271-96$

16 Spielberg C, Tanczos P, Buchwalsky R. New experience in the diagnosis of sick sinus syndrome. $Z$ Kardiol 1977;66: $374-81$

17 Reiffel JA, Bigger JT, Cramer M, et al. Ability of Holter electrocardiographic recording and atrial stimulation to detect sinus nodal dysfunction in symptomatic and asymptomatic patients with sinus bradycardia. Am 7 Cardiol 1977;40:189patient.

18 Szatmáry L, Jouve A, Pinot JJ, et al. Comparative study of electrophysiological and Holter monitoring data in estimating sinoatrial function. Cardiology 1983;70:184-93.

19 Kulbertus H, de Leval-Rutten F, Demoulin J. Sino-atrial disease: a report of 13 cases. 7 Electrocardiol 1973;6:303-12.

20 Fromer M, Kappenberger L, Steinbrunn W. Binodal disease: diseased sinus node and atrioventricular block. Z Cardiol 1983;72:410-13.

21 Rakovec P. Sinus node recovery time in patients with complete atrioventricular block. Cardiology 1981;67:31-7.

22 Vardas PE, Fitzpatrick A, Ingram A. Natural history of sinus node chronotropy in paced patients. PACE 1991;14:155-

23 Gwinn N, Leman R, Kratz J, et al. Chronotropic incompetence: a common and progressive finding in pacemaker patients. Am Heart f 1992;123:1216-19.

24 Ellestad MH, Wan MV. Predictive implications of stress testing. Follow-up of 2700 subjects after maximal treadmill stress testing. Circulation 1975;51:363-9.

25 Andersen HR, Thuesen L, Bagger JP, et al. Prospective randomised trial of atrial versus ventricular pacing in sick-sinus syndrome. Lancet 1994;344:1523-8.

26 Levander-Lindgren M, Pehrsson SK. Occurrence and significance of arrhythmias associated with atrial-triggered ventricular pacing. PACE 1984;7:628-39.

27 Gross IN, Moser S, Benedek ZM, et al. DDD pacing mode survival in patients with a dual-chamber pacemaker. $7 \mathrm{Am}$ Coll Cardiol 1992;19:1536-41.

28 Ibrahim B, Sanderson JE, Wright B, et al. Dual chamber pacing: how many patients remain in DDD-mode over a long term? Br Heart f 1995; 74:76-9.

29 Nowak B, Voigtlaender T, Rosocha S, et al. High degree AV block and paroxysmal atrial fibrillation. An indication for single lead $\operatorname{VDD}(\mathrm{R})$ pacing with mode-switching? [abstract] PACE 1997;20:1169.

30 Vaziri SM, Larson MG, Benjamin EJ, et al. Echocardiographic predictors of nonrheumatic atrial fibrillation. The Framingham heart study. Circulation 1994;89:724-30.

31 Lauer MS, Okin PM, Larson MG, et al. Impaired heart rate response to graded exercise. Prognostic implications of chronotropic incompetence in the Framingham heart study. Circulation 1996;93:1520-6. 